\title{
Syncytia formation during SARS-CoV-2 lung infection: a disastrous unity to eliminate lymphocytes
}

\author{
Liangyu Lin $\mathbb{D}^{1} \cdot$ Qing $\mathrm{Li}^{1} \cdot$ Ying Wang $\mathbb{D}^{1} \cdot$ Yufang Shi ${ }^{1,2,3}$
}

Received: 18 April 2021 / Revised: 21 April 2021 / Accepted: 21 April 2021 / Published online: 12 May 2021

(c) The Author(s), under exclusive licence to ADMC Associazione Differenziamento e Morte Cellulare 2021

Coronavirus disease 2019 (COVID-19), caused by the severe acute respiratory syndrome coronavirus 2 (SARS$\mathrm{CoV}-2$ ) infection, has become a major threat to the global public health and economy [1,2]. While the majorities of SARS-CoV-2 infected individuals only suffer mild or moderate symptoms, unfortunately, some patients develop severe chronic respiratory diseases (CRDs) and have to be admitted to intensive care units [3, 4]. When the lungs of CRDs patients were analyzed histologically, many of them possess multinucleate pneumocytes. Two independent studies from the Mauro Giacca group and the Qiang Sun group, which were just published in Nature and Cell Death and Differentiation, provided critical insights into the causes and consequences of syncytia during SARS-CoV-2 infections $[5,6]$.

Syncytia are evolutionarily conserved cellular structures form by the multiple cell fusions of uninuclear cells. In mammals, the best example of physiological syncytia is muscle fibers, which contain thousands of fused muscle cells to allows their rapid coordinated contraction [7]. It is

Supplementary information The online version contains supplementary material available at https://doi.org/10.1038/s41418021-00795-y.

Ying Wang

yingwang@sibs.ac.cn

$\triangle$ Yufang Shi

yfshi@suda.edu.cn

1 CAS Key Laboratory of Tissue Microenvironment and Tumor, Shanghai Institute of Nutrition and Health, University of Chinese Academy of Sciences, Chinese Academy of Sciences, Shanghai, China

2 The First Affiliated Hospital of Soochow University, State Key Laboratory of Radiation Medicine and Protection, Institutes for Translational Medicine, Soochow University Medical College, Suzhou, Jiangsu, China

3 Department of Experimental Medicine, TOR, University of Rome Tor Vergata, Rome, Italy also important in the decidualization process during embryo implantation [8]. Syncytia can also be induced by certain types of infections by viruses, such as human immunodeficiency virus, respiratory syncytial virus, and herpes simplex virus [9]. It could be envisioned that virus-induced cell fusion facilitates the transfer of viral genomes to the neighboring cells. However, the viral and cellular mechanisms regulating the formation of syncytia during SARS-CoV-2 infection remains largely elusive.

While examining the histopathologic lung sections from patients died from COVID-19, the Giacca group and the Sun group observed the prevailing existence of atypical cells containing 2-20 nuclei. The identities of these syncytia were later confirmed by their expression of pneumocyte specific makers. In vitro co-culture assay showed that monkey kidney epithelial cell line, Vero cells $\left(\mathrm{ACE}^{+}\right)$, upon expressing the SARS-CoV-2 spike protein, could form homologous syncytia or fuse with other cell lines as long as the ACE2 receptor was present. Interestingly, when Vero cells were transfected with Spike protein from SARSCoV-1, no formation of syncytia was observed. Therefore, the key element responsible for SARS-CoV-2-mediated syncytia is absent in the spike protein of SARS-CoV-1. Driven by this hypothesis, Sun and et al. compared the spike protein from SARS-CoV-2 and SARS-CoV1 and found that there is a four amino acids (PRRA) insertion before the S1/S2 cleavage site in the SARS-CoV-2 spike protein. The truncated mutation of SARS-CoV-2 spike protein with "PRRA" deletion lose its abilities to fuse cells. Consistently, spike protein from SARS-CoV1 effectively induced syncytia once the "PRRA" sequence was inserted before the S1/S2 cleavage site of the SARS-CoV-1 genome. Furthermore, the Sun group demonstrated that a bi-arginine motif containing R682 and R685 dictates syncytium formation by constructing single or combine mutations in the "PPRA" insertion. Fig. 1.

The new data obtained by Sun et al. provide critical information for understanding syncytia through deciphering the structure basis required for SARS-CoV-2 spike protein- 
Fig. 1 The SARS-CoV-2 spike protein and cellular TMEM16 ion channel collaboratively mediated the formation of syncytia in COVID-19 infections. Pneumocytes infected with SARS-CoV-2 expressed the virus spike protein on their surface membrane. The bi-arginine motif (R682 and R685) in the spike protein interacted with ACE2 on health cells and activated TMEM16F, which eventually induced cell fusion. The syncytia formation on one hand facilitated virus transmission, and on the other hand, endowed multinucleate pneumocytes the abilities to engulf $\mathrm{CD} 45^{+}$cells.

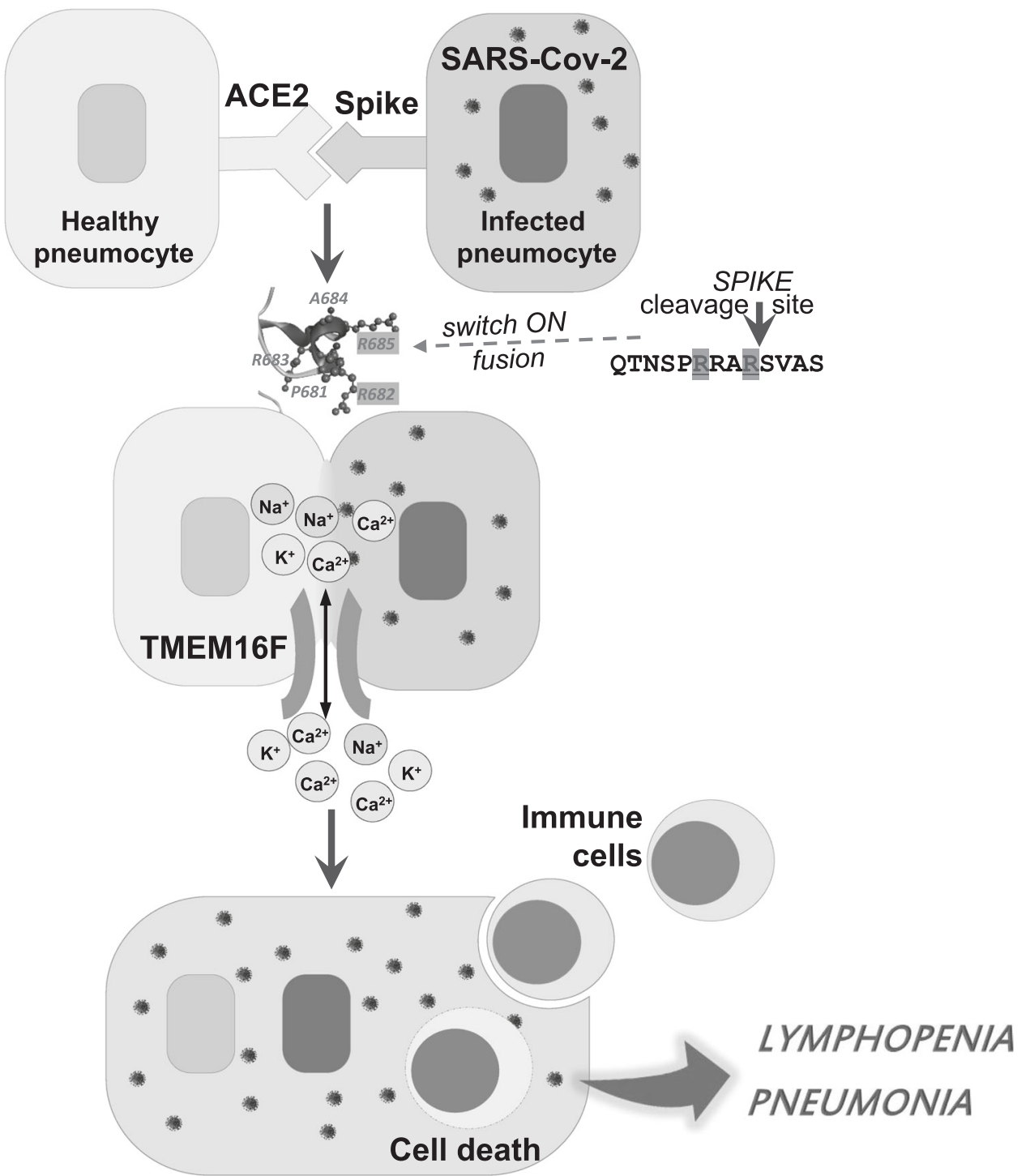

mediated cell fusion, while Giacca et al. focused on the cellular mechanism and therapeutic potential of syncytia during SARS-Cov-2 infection. In this regard, Giacca and colleagues screened 3049 FDA/EMA-approved drugs using SARS-CoV-2 spike protein expressing Vero cell-based in vitro cell fusion system to search for drugs that block syncytia. Interestingly, drugs that suppressed cell fusion are all capable of regulating intracellular $\mathrm{Ca}^{+}$levels. Among the syncytia blocking drugs, niclosamide, an oral antihelminthic agent, was found to be effective at a very low dose $(\mathrm{IC} 50=0.34 \mu \mathrm{M})$ and could prevent cell from virusinduced cell death. Niclosamide is a potent antagonist of $\mathrm{Ca} 2^{+}$-activated TMEM16/anoctamin family of chloride channels [10]. TMEM16F was also dramatically increased in Vero cells upon spike protein expression. When TMEM16F expression was disturbed, the syncytia formation in spikeexpression cells were diminished. Therefore, TMEM16F activation is the signal responsible for triggering syncytia.
These two elegant studies collectively revealed a new concept of syncytia formation and its roles in SARS-CoV-2 infections, which can be briefly précised as follows. The SARS-CoV-2 infections induce the surface expression of the spike glycoprotein. The interaction of the spike protein with the ACE2 receptor of the neighboring cells then activate TMEM16F, and trigger the unshealthe of the profusion S2 fragment of the spike protein in a bi-arginine motif dependent manner, which eventually leads to the membrane fusion and syncytia formation. However, there are still many questions that remain to be elucidated. One of which is whether the biarginine motif is required for the activation of TMEM16F. Another is the impact of syncytia formation on SARS-CoV-2 infections in vivo. Sun et al. found that a type of CD45 positive cell structure presents in the syncytia of the COVID-19 patients. This could be a cell-in-cell structure. When human peripheral blood mononuclear cells were co-cultured with SARS-CoV-2 spike protein-induced syncytia, they could be 
engulfed by and die inside the syncytia, thus providing a possible explanation for lymphopenia in SARS-CoV-2 infections [11]. It can be highly suspected that syncytia are deleterious for COVID-19 patients since syncytia were observed only in the severe stages of the diseases and syncytia may induce lymphopenia. Despite the observation of multinucleate pneumocytes in autopsy, it is still not known whether such syncytia play a critical role in the pathogenesis of CRDs of severe COVID-19 patients. Recently, an antidepressant drug, fluvoxamine, was shown to lower the likelihood of clinical deterioration of severe COVID-19 patients in a randomized clinical trial [12]. Interestingly, fluvoxamine could facilitate TMEMF activation and phosphatidylserine exposure [13]. It is imperative to examine whether fluvoxamine affects syncytia formation. It is also worthy to evaluate the whether the combine uses of anti-syncytia drugs with other COVID-19 targets would yield better clinical outcomes [14, 15].

Overall, these two papers provide critical information for the understanding of how syncytia occurred during SARS$\mathrm{CoV}-2$ infections at the virus structure and cellular signaling points of views and open up a new revenue in COVID-19 studies. It is anticipated that these novel findings may provide information for developing new strategies to combat the current pandemic.

Acknowledgements We thank Gerry Melino for his insightful suggestions on the paper and help on editing the figure.

Author contributions Conceptualization, LYL and QL; Figures, QL; Writing-Original Draft, LYL and QL; Writing-Review \& Editing, YFS and YW; Supervision, YFS and YW.

Funding The work has been partially supported by grants from the National Key R\&D Program of China (2018YFA0107500), the Scientific Innovation Project of the Chinese Academy of Sciences (XDA16020403), Suzhou 2020 Emergency Innovation Funding on COVID-19 Infection, the National Natural Science Foundation of China (81861138015, 81530043, 31961133024, 32000626, 31771641,81930085 and 81571612 ). Fellowship of China Postdoctoral Science Foundation (2020M671261).

\section{Compliance with ethical standards}

Conflict of interest The authors declare no competing interests.

Ethical approval Our study did not require ethical approval.
Publisher's note Springer Nature remains neutral with regard to jurisdictional claims in published maps and institutional affiliations.

\section{References}

1. Zhu N, Zhang D, Wang W, Li X, Yang B, Song J, et al. A Novel Coronavirus from Patients with Pneumonia in China, 2019. N Engl J Med. 2020;382:727-33.

2. Chen J, Lu H, Melino G, Boccia S, Piacentini M, Ricciardi W, et al. COVID-19 infection: the China and Italy perspectives. Cell Death Dis. 2020;11:438.

3. Shi Y, Wang Y, Shao C, Huang J, Gan J, Huang X, et al. COVID19 infection: the perspectives on immune responses. Cell Death Differ. 2020;27:1451-4.

4. Forni G, Mantovani A, Covid-19 Commission of Accademia Nazionale dei Lincei R. COVID-19 vaccines: where we stand and challenges ahead. Cell Death Differ. 2021;28:626-39.

5. Braga L, Ali H, Secco I, Chiavacci E, Neves G, Goldhill D, et al. Drugs that inhibit TMEM16 proteins block SARS-CoV-2 Spikeinduced syncytia. Nature. 2021.

6. Zhang ZY, Zheng Y, Niu Z, Zhang B, Wang C, Yao X, et al. A bi1 Arginine Motif in the SARS-CoV-2 Spike Protein Dictates Syncytium-mediated Lymphocyte Elimination via Cell-in-Cell Structures. Cell Death Differ. 2021.

7. Lee DM, Chen EH. Drosophila Myoblast Fusion: invasion and Resistance for the Ultimate Union. Annu Rev Genet. 2019;53:67-91.

8. Wang H, Dey SK. Roadmap to embryo implantation: clues from mouse models. Nat Rev Genet. 2006;7:185-99.

9. Leroy H, Han M, Woottum M, Bracq L, Bouchet J, Xie M, et al. Virus-Mediated Cell-Cell Fusion. Int J Mol Sci. 2020;21;9644.

10. Miner K, Labitzke K, Liu B, Wang P, Henckels K, Gaida K, et al. Drug Repurposing: the Anthelmintics Niclosamide and Nitazoxanide Are Potent TMEM16A Antagonists That Fully Bronchodilate Airways. Front Pharm. 2019;10:51.

11. Agrati C, Sacchi A, Bordoni V, Cimini E, Notari S, Grassi G, et al. Expansion of myeloid-derived suppressor cells in patients with severe coronavirus disease (COVID-19). Cell Death Differ. 2020;27:3196-207.

12. Lenze EJ, Mattar C, Zorumski CF, Stevens A, Schweiger J, Nicol $\mathrm{GE}$, et al. Fluvoxamine vs Placebo and Clinical Deterioration in Outpatients With Symptomatic COVID-19: a Randomized Clinical Trial. JAMA. 2020;324:2292-300.

13. Kim HJ, Jun I, Yoon JS, Jung J, Kim YK, Kim WK, et al. Selective serotonin reuptake inhibitors facilitate ANO6 (TMEM16F) current activation and phosphatidylserine exposure. Pflug Arch. 2015;467:2243-56.

14. Novelli G, Liu J, Biancolella M, Alonzi T, Novelli A, Patten JJ, et al. Inhibition of HECT E3 ligases as potential therapy for COVID-19. Cell Death Dis. 2021;12:310.

15. Matsuyama T, Kubli SP, Yoshinaga SK, Pfeffer K, Mak TW. An aberrant STAT pathway is central to COVID-19. Cell Death Differ. 2020;27:3209-25. 